\title{
Bipolar hemiarthroplasty of hip joint: prospective randomised comparative study of direct anterior approach versus posterior approach
}

\author{
Mukesh Sancheti ${ }^{1}$, Mahendra Ghagre ${ }^{2 *}$
}

\author{
${ }^{1}$ Department of Orthopaedics, Kingsway Hospitals, Nagpur, Maharashtra, India \\ ${ }^{2}$ Department of Orthopaedics, Dr Ghagre Hospital, Karanja (G), Maharashtra, India
}

Received: 01 January 2021

Accepted: 03 February 2021

\section{*Correspondence:}

Dr. Mahendra Ghagre,

E-mail: drmahendraghagre@ rediffmail.com

Copyright: (C) the author(s), publisher and licensee Medip Academy. This is an open-access article distributed under the terms of the Creative Commons Attribution Non-Commercial License, which permits unrestricted non-commercial use, distribution, and reproduction in any medium, provided the original work is properly cited.

\section{ABSTRACT}

Background: The dilemma while treating cases with hemiarthroplasty is the surgical approach to be employed. The aim of present study is comparison of posterior approach and direct anterior approach with regards to various relevant outcomes and evaluate early surgical complications.

Methods: In this randomized observational study conducted over 2 years, 20 patients each with intra-capsular neck femur fracture were enrolled as per predefined selection criteria and treated with hemiarthroplasty (bipolar) either by posterior approach or by direct anterior approach. Follow up was done for all the patients at regular intervals with standard post-operative protocol, including evaluation for surgical complications, active range of motion and assessment of Harris hip score.

Results: Overall infection rate was 5\% (two patients in posterior approach, no patient in direct anterior approach, the difference being statistically insignificant). No incidences of dislocation were reported in either group. Two patients of posterior approach had abductor weakness. Periprosthetic fracture and deep vein thrombosis were observed in one case each in posterior approach group. In posterior surgical group, excellent Harris hip score in 25\% patients, good in $57.5 \%$ patients and fair in $17.5 \%$ patients were observed; while in direct anterior surgical group, excellent score was observed in $12.5 \%$ patients, good in $55 \%$ patients and fair in $32.5 \%$ patients.

Conclusions: No major clinically or statistically significant differences were observed between for outcomes and complications between the two approaches. Larger randomized trials with bigger samples are recommended.

Keywords: Fracture femur neck, Hemiarthroplasty, Direct anterior approach, Posterior approach

\section{INTRODUCTION}

Hip fractures are common and comprise about $20 \%$ of the operative workload of an orthopaedic trauma unit. ${ }^{1}$ Lifetime risk of sustaining a hip fracture is high and lies within the range of 40 to $50 \%$ in women and 13 to $22 \%$ in men. ${ }^{1}$ With the increase in life expectancy worldwide and the prevalence of osteoporosis going up, the number of hip fractures is expected to increase from 1.66 million in 1990 to 6.26 million in 2050 worldwide. $^{2}$
Femoral neck fracture is common in osteoporotic, old individuals. Management of displaced fracture neck of femur has been one daunting task for the orthopaedic surgeons; and internal fixation, hemiarthroplasty and total hip arthroplasty are the various treatment options for such fractures. There has been some ambiguity with respect to the appropriate management in young/middle aged adults as to whether to opt for head preserving procedure or arthroplasty. ${ }^{3}$ Hemiarthroplasty/total arthroplasty does have a definitive place in management of displaced femoral neck fractures in older subjects. Hemiarthroplasty 
has many advantages since it allows immediate return to daily activities and avoids bed rest related complications. The procedure carries a relatively shorter duration of surgery with reasonable clinical outcomes. ${ }^{4,5}$ One important issue while treating patients with hemiarthroplasty is the type of surgical approach. Two different surgical approaches have predominated; the direct anterior approach and the posterior approach. Recently some less invasive modifications have been described and compared to the standard approaches such as Kocher-Langenbeck (dorsal), Bauer or Hardinge (lateral), Watson-Jones (anterolateral), and SmithPeterson (anterior) etc. ${ }^{5}$ All of them seem to have some advantages and every modification leads to new problems; prompting the orthopedic surgeons to reconsider traditional anterior or posterior approaches only. ${ }^{5}$ It is still unclear whether one approach may be advantageous; although lower dislocation rates after the direct anterior approach have been documented. ${ }^{6}$

The aim of this study is to compare the posterior approach and the direct anterior approach with regards to various primary and secondary outcomes and evaluate early surgical complications in patients being treated with bipolar hemiarthroplasty after femoral neck fracture.

\section{METHODS}

This is a prospective, comparative, randomized observational study comprising of patients with intracapsular neck femur fracture treated with hemiarthroplasty (bipolar) by posterior or direct anterior approach. The study was conducted from October 2015 to October 2017 ( 2 years) by the department of orthopedics at a tertiary care government hospital in central India.

Following selection criteria were adopted for the study:

\section{Inclusion criteria}

Inclusion criteria included all patients with age above 50 years and below 80 years, painless hip before trauma, no other associated hip pathology and patients who are fit and willing to go for surgery.

\section{Exclusion criteria}

Exclusion criteria excluded intertrochanteric femur fractures, pathological neck femur fractures, patients with an additional acute lower extremity fracture in addition to the neck femur fracture, intra-capsular neck femur fracture managed by any other approach or treatment modality, neurological deficit involving same limb, patients with severe medical comorbidities/unfit for surgery, revision surgery and not willing to consent for the study.

A total of 40 patients were enrolled in the study as per mentioned selection criteria and operated upon either by posterior approach or by direct anterior approach (20 participants each). The randomization was achieved using simple random sampling method.

\section{Treatment protocol}

The patients were admitted as emergencies with an even distribution under care of various consultants as per unit allocation. Detailed history and thorough clinical examination were undertaken. General condition of the patient including vitals, associated injuries and lifethreatening injuries were assessed. Local examination including swelling, tenderness, deformity and distal neurovascular status were assessed. On admission ankle traction with an appropriate weight was employed to relieve pain and reduce shortening. Radiological examination including antero-posterior and lateral view of the injured hip was taken. A routine blood investigation for anesthesia fitness was done. Patients were operated after taking written informed consent.

The patients were divided by simple random sampling in two groups: group A: patients operated by posterior approach and group B: patients operated by direct anterior approach.

Patients of both the surgical groups were followed through same standard post-operative protocol. Regular follow up was done for all the patients at 1, 3 and 6 months. X-rays were done at each follow-up. Patients were evaluated for surgical complications like dislocation, infection, postoperative bleeding or hematoma, peri-prosthetic fractures at each follow up visit. Active range of motion was assessed along with assessment of Harris hip score. ${ }^{7}$

\section{Statistical methods}

Data analysis was performed using SPSS (version 18). The continuous variables were handled by calculating the mean and standard deviation. The significance of difference between two continuous variables was calculated by applying student's $t$ test and $p$ value $>0.05$ was considered statistically significant arbitrarily.

The study was started only after taking necessary approval from the institutional ethics committee and all the patients gave written informed consent for participation.

\section{RESULTS}

A total of 40 patients with intra-capsular neck femur fracture were operated upon, either by posterior approach or by direct anterior approach for bipolar hemiarthroplasty (20 participants each). Mean age of the participants was $64.60 \pm 6.88$ years for posterior approach and $60.75 \pm 7.10$ years for direct anterior approach $(\mathrm{p}>0.05)$; while gender distribution was also similar $(\mathrm{p}>0.05)$ by design. Both right and left sides were almost equally involved in across both the categories. Fall was the commonest mode of trauma in the study (19 out of 20 in posterior approach group and 18 out of 20 in direct anterior group). The distribution of patients as per fracture type in the two surgical approaches group was almost even as an effect of randomization (Table 1). 
Table 1: Distribution of patients in two groups according to the fracture type.

\begin{tabular}{|lll|}
\hline Type of fracture & $\begin{array}{l}\text { Posterior } \\
\text { approach }\end{array}$ & $\begin{array}{l}\text { Direct anterior } \\
\text { approach }\end{array}$ \\
\hline Basicervical (BC) & 4 & 3 \\
\hline Subcapital (SC) & 11 & 10 \\
\hline Transcervical (TC) & 5 & 7 \\
\hline
\end{tabular}

The differences between operative parameters of two approaches were studied by applying t-test for independent samples. The difference between mean operative times of surgery of two approaches was found to be statistically significant. The intraoperative blood loss was significantly lower in direct anterior approach. Overall infection rate in all the patients operated for hemiarthroplasty was 5\%. Two patients in posterior approach and no patient in direct anterior approach got infected, the difference being statistically insignificant. No incidences of dislocation were reported in either group. Abductor weakness was present in two patients of posterior approach, while no patient operated with direct anterior approach showed abductor weakness (Table 2).

Table 2: Perioperative parameters comparison between two approaches.

\begin{tabular}{|llll|}
\hline & $\begin{array}{l}\text { Posterior } \\
\text { approach } \\
\text { Parameter } \\
\text { 2SD) }\end{array}$ & $\begin{array}{l}\text { Direct } \\
\text { anterior } \\
\text { approach } \\
\text { (Mean } \pm\end{array}$ & P \\
2SD) & \\
\hline $\begin{array}{l}\text { Mean operative } \\
\text { time (min) }\end{array}$ & $\begin{array}{l}79.0 \pm \\
10.20\end{array}$ & $\begin{array}{l}115.5 \pm \\
7.93\end{array}$ & $<0.001$ \\
\hline $\begin{array}{l}\text { Intra operative } \\
\text { blood loss (ml) }\end{array}$ & $140.0 \pm$ & $111.0 \pm$ & $<0.001$ \\
$\begin{array}{l}\text { Overall } \\
\text { infection rate } \\
\text { (\%) }\end{array}$ & 10 & 11.30 & \\
\hline $\begin{array}{l}\text { Dislocation } \\
\text { Abductor }\end{array}$ & 0 & 0 & $>0.05$ \\
weakness & 2 & 0 & $>0.05$ \\
\hline
\end{tabular}

Other surgical complications such as periprosthetic fracture and deep vein thrombosis were observed in one case each in posterior approach group; while sciatic nerve injury, periprosthetic femur fracture, intraoperative or perioperative mortality, aseptic loosening of prosthesis, erosion of acetabulum, deep vein thrombosis and pulmonary embolism were not noted in any of the case operated by direct anterior group (Table 3 ).

Secondary procedures were done to manage the complication of periprosthetic fracture in one patient with posterior approach group under spinal anesthesia. No patient in direct anterior group required revision surgery. One patient had right ipsilateral proximal humerus fracture along with neck femur fracture in direct anterior approach surgical group which was managed conservatively by immobilization for 1 month by universal shoulder immobilizer. No patient had any associated injury in posterior surgical approach group.

Table 3: Other surgical complications in both groups.

\begin{tabular}{|lll|}
\hline Complications & $\begin{array}{l}\text { Posterior } \\
\text { approach } \\
(\mathbf{n}=20)\end{array}$ & $\begin{array}{l}\text { Direct anterior } \\
\text { approach } \\
(\mathbf{n}=20)\end{array}$ \\
\hline Sciatic nerve injury & 0 & 0 \\
\hline $\begin{array}{l}\text { Periprosthetic } \\
\text { fracture }\end{array}$ & 1 & 0 \\
\hline Mortality & 0 & 0 \\
\hline Aseptic loosening & 0 & 0 \\
\hline Acetabular erosion & 0 & 0 \\
\hline $\begin{array}{l}\text { Deep vein } \\
\text { thrombosis }\end{array}$ & 1 & 0 \\
\hline $\begin{array}{l}\text { Pulmonary } \\
\text { embolism }\end{array}$ & 0 & 0 \\
\hline
\end{tabular}

The functional outcome of hip after hemiarthroplasty was assessed with Harris hip score. In our study in posterior surgical group, we had excellent score in $25 \%$ patients, good in $57.5 \%$ patients and fair in $17.5 \%$ patients. In direct anterior surgical group, we had excellent score in $12.5 \%$ patients, good in $55 \%$ patients and fair in $32.5 \%$ patients. No patients in both the approaches had poor result. The average Harris hip scores at end of 1, 3 and 6 months follow up periods were statistically comparable between the groups (Table 4).

Table 4: Comparison of Harris hip score between two groups at different follow up periods.

\begin{tabular}{|lll|}
\hline $\begin{array}{l}\text { Timeline } \\
\text { (month) }\end{array}$ & $\begin{array}{l}\text { Posterior } \\
\text { approach }\end{array}$ & $\begin{array}{l}\text { Direct anterior } \\
\text { approach }\end{array}$ \\
\hline $\mathbf{1}$ & $66.17 \pm 7.68$ & $66.49 \pm 9.96$ \\
\hline $\mathbf{3}$ & $74.51 \pm 7.31$ & $76.51 \pm 7.07$ \\
\hline $\mathbf{6}$ & $82.98 \pm 6.10$ & $85.98 \pm 6.13$ \\
\hline
\end{tabular}

\section{DISCUSSION}

The main aim of the present study was to evaluate and compare the effects of posterior and direct anterior surgical approaches in hemiarthroplasty as treatment modality for intra-capsular neck femur fractures cases.

The difference for average mean age and the sex distribution was insignificant as an effect of randomization. Both right and left sides were almost equally involved across the categories. Low energy injuries which include fall at home, fall from bed, slip in bathroom were the most common cause of fracture as compared to high energy injuries like road traffic accidents. The distribution between two surgical approaches was almost same with 95 and 90\% low energy injuries in posterior and direct anterior approach respectively; which is in line with most of the previous studies and nearly comparable to observations of Biber and Parker et al. ${ }^{8,9}$ 
The primary operative parameters were compared for understanding superiority of one approach over the other. The mean operating time for posterior approach was significantly lower in the present study. Very few studies have mentioned the operating time in minutes; viz. Keene, Parker and Jalovaara et al. 6,9,10 The operating time for posterior surgical group as well as the difference observed in our study was comparable to these studies although the difference was observed to be insignificant in the study by Parker et al. ${ }^{9}$ The intraoperative blood loss was observed to be significantly higher in posterior approach in the present study. Again, very few studies have studied intraoperative blood loss as an outcome. ${ }^{6}$ Both the above parameters are person dependent and may vary between surgeons. Statistically insignificant difference was observed between the two approaches for postoperative infection rate. The rate of infection was reported to be slightly higher in posterior surgical approach in studies done by Parker and Mukka et al, while it was insignificantly lower in the study by Biber et al. ${ }^{8,9,11}$ The overall infection rate in all patients operated for hemiarthroplasty irrespective of the approach was observed at $5 \%$ in our study which is quite high as compared to previous similar studies. It is believed that the approach used does not affect the infection rate. There are many risk factors predisposing to infection such as comorbidities of patients, poor patient hygiene and low sterile conditions etc. The infection rate may be brought down with cautious, sterile operating conditions, proper pre- and post-operative antibiotic administration and, probably, with the use of cement loaded with antibiotics. Further elaborate, adequately powered studies may be required to test these assumptions. No patients operated by posterior approach or anterior approach had suffered dislocation in the present study; probably due to meticulous posterior capsule and external rotators repair. Most of the previous studies indicate that the posterior approach carries an increased risk of prosthetic dislocation. In the studies done by Kwon and Bush et al, posterior approach had been reported to be associated with 4.46 and $4.5 \%$ dislocation rate respectively. ${ }^{12,13}$ Abductor weakness was observed in two cases in posterior approach group in the present study and was thought to be due to direct surgical trauma to the muscle attachment to greater trochanter, while no patient operated with direct anterior approach had it. The two patients' clinical outcome didn't deteriorate owing to proper physiotherapy rehabilitation program. Further, the posterior approach at times results in delayed regain of function as there is more damage to the hip muscles.

In other postoperative complications, there was one incidence each of peri-prosthetic fracture and one deep vein thrombosis in posterior approach group. No other postoperative complications like sciatic nerve injury, aseptic loosening of prosthesis, acetabular erosion, pulmonary embolism or mortality were observed in either surgical group. In the study done by Keene et al, $1 \%$ of those operated with posterior approach developed sciatic nerve injury. ${ }^{6}$ Parker et al reported $1.9 \%$ patients having developed sciatic nerve palsy and peri-prosthetic fracture in the posterior surgical approach group, while $0.9 \%$ patients developed deep vein thrombosis in both the groups..$^{9}$ In a study done by Mukka et al, peri-prosthetic fracture developed in $1 \%$ patients operated with direct anterior approach while acetabular erosion was seen in $1 \%$ patients operated with posterior approach. ${ }^{11}$ Thus, the rate of postoperative complications viz. peri-prosthetic fracture and deep vein thrombosis was relatively higher in the present study for no obvious reasons.

In most previously similar studies on intracapsular neck femur fracture the functional outcome has been assessed by modified Harris hip score; as was done in the present study. Patients in both groups had good average Harris hip score and the difference was statically insignificant. Distribution of the score in present study is comparable to studies performed by Parker and Mukka et al. ${ }^{9,11}$

One patient underwent secondary procedure in the form of ORIF with encircle with SS wire in a case of peri-prosthetic fracture in the posterior group. No patient in direct anterior group required revision surgery. The requirement of secondary procedure in the present study is similar to that of Parker et al. ${ }^{9}$ In most studies it had been performed for infection with debridement, dislocation with open reduction and peri-prosthetic fracture with plating. $., 12,13$

\section{CONCLUSION}

In conclusion, it can be said that there were no major statistically or clinically significant differences in outcomes and complications between the two approaches. Thus, recommendation for a certain surgical approach remains an expert opinion based on subjective judgement on the severity of observed complications. The quality of literature regarding the surgical approaches is limited. Larger randomized trials may further improve our insight and are recommended.

\section{Funding: No funding sources \\ Conflict of interest: None declared}

Ethical approval: The study was approved by the institutional ethics committee

\section{REFERENCES}

1. Singer BR, McLauchlan GJ, Robinson CM, Christie J. Epidemiology of fractures in 15000 adults: the influence of age and gender. $\mathrm{J}$ bone joint surg. Bri. 1998;80(2):243-8.

2. Dennison E, Mohamed MA, Cooper C. Epidemiology of osteoporosis. Rheumatic Dis Clin. 200632(4):61729.

3. Gautam VK, Anand S, Dhaon BK. Management of displaced femoral neck fractures in young adults (a group at risk). Injury. 1998;29(3):215-8. 
4. Zofka P. Bipolar hip hemiarthroplasty. Acta Chirurgiae Orthopaedicae et Traumatologiae Cechoslovaca. 2007;74(2):99-104.

5. Auffarth A, Resch H, Lederer S, Karpik S, Hitzl W, Bogner R et al. Does the choice of approach for hip hemiarthroplasty in geriatric patients significantly influence early postoperative outcomes? A randomized-controlled trial comparing the modified Smith-Petersen and Hardinge approaches. J Trauma Acute Care Surg. 2011;70(5):1257-62.

6. Keene GS, Parker MJ. Hemiarthroplasty of the hipthe anterior or posterior approach? A comparison of surgical approaches. Injury. 1993;24(9):611-3.

7. Malchau $\mathrm{H}$, Soderman $\mathrm{P}$, Herberts $\mathrm{P}$. The validity and reliability of Harris Hip Score. Read SICOT. 1999:1823.

8. Biber R, Brem M, Singler K, Moellers M, Sieber C, Bail HJ. Dorsal versus transgluteal approach for hip hemiarthroplasty: an analysis of early complications in seven hundred and four consecutive cases. Int orthop. 2012;36(11):2219-23.

9. Parker MJ. Hemiarthroplasty versus internal fixation for displaced intracapsular fractures of the hip in elderly men: a pilot randomised trial. Bone Joint J. 2015;97(7):992-6.
10. Jalovaara P, Virkkunen H. Quality of life after primary hemiarthroplasty for femoral neck fracture: 6-year follow-up of 185 patients. Acta Orthop Scandinavica. 1991;62(3):208-17.

11. Mukka S, Mahmood S, Kadum B, Sköldenberg O, Sayed-Noor A. Direct lateral vs posterolateral approach to hemiarthroplasty for femoral neck fractures. Orthop Traumatol Surg Res. 2016;102(8):1049-54.

12. Kwon MS, Kuskowski M, Mulhall KJ, Macaulay W, Brown TE, Saleh KJ. Does surgical approach affect total hip arthroplasty dislocation rates? Clin Orthop Rel Res. 2006;447:34-8.

13. Bush JB, Wilson MR. Dislocation after hip hemiarthroplasty: anterior versus posterior capsular approach. Orthopedics. 2007;30(2):138-44.

Cite this article as: Sancheti M, Ghagre M. Bipolar hemiarthroplasty of hip joint: prospective randomised comparative study of direct anterior approach versus posterior approach. Int J Res Orthop 2021;7:381-5. 